\title{
ОЖЕ-РЕКОМБИНАЦИЯ В ПОЛУПРОВОДНИКАХ
}

М. Н. Аликулов, кандидат физико-математических наук, доиент кафедры физики, Каршинский инженерно-экономический институт, г. Карши, Узбекистан

\section{DOI: https://doi.org/10.31435/rsglobal_conf/30052021/7575}

Abstract. The Auger recombinations occurring in semiconductors have been studied in this work. The theoretical and analytical data of Auger recombination processes in semiconductors with straight and complex bands are highlighted. The relationship between the rate of recombination and the structure of the bands of semiconductors has been studied. Expressions of the laws of conservation of energy and momenta are given for the processes of recombination of semiconductors with straight bands.

Keywords: silicon, recombination, energy, momentum, impurity, semiconductor, lifetime, electron, hole, concentration

Изучение процессов рекомбинации важно для понимания процессов, происходящих в полупроводниках и полупроводниковых приборах. На сегодняшний день широко изучены три типа рекомбинации [1-6]. Это излучательная рекомбинация, Оже-рекомбинация или ударная рекомбинация и рекомбинация Шокли-Рида-Холла через глубокие поверхности.

Излучательная рекомбинация - это процесс аннигиляции пары электрон-дырка, при котором выделяемая энергия излучается в виде фотона.

Оже- или ударная рекомбинация - это трехчастичный процесс, в котором энергия, выделяющаяся при аннигиляции пары электрон-дырка, передается третьей частице.

В рекомбинации Шокли-Рида-Холла процессы рекомбинации в полупроводниках происходят через примесные центры.

В работе [6] рассмотрены основные механизмы Оже-рекомбинации. На основе модели Битти-Ландсберга-Блэкмора автором исследованы зависимость скорости и времени жизни генерации-рекомбинации от состава и температуры $\mathrm{HgCdTe}$ материала типа n- и p-.

В работе [7] проведен расчет зависимости концентрации носителей заряда, участвующих в излучательной рекомбинации от интенсивности накачки.

В работе автора [8] изучена зависимость рекомбинационных процессов, происходящих в полупроводниках, от структуры полупроводниковых зон. Обоснованы преимущества использования непрямых зональных полупроводников при создании солнечных батарей.

В данной статье представлены теоретические и аналитические данные о процессах Ожерекомбинации в полупроводниках и вытекающие из них выводы.

В процессах Оже-рекомбинации в полупроводниках n-типа участвуют два электрона и одна дырка, а в полупроводниках р-типа участвуют две дырки и один электрон. Скорость Ожерекомбинации равна

$$
r_{n}=c_{n} n^{2} p, \quad r_{p}=c_{p} p^{2} n
$$

Из этого выражения видно, что скорость рекомбинации зависит от концентрации электронов и дырок. Наряду с этим скорость рекомбинации в полупроводниках также зависит от структуры полупроводниковых зон.

Сначала рассмотрим процессы рекомбинации, происходящие в прямозонных полупроводниках (рис. 1а).

Известно, что законы сохранения энергии и импульса справедливы для всех процессов, происходящих в полупроводниках. Законы сохранения энергии и импульса для этого процесса следующие.

$\vec{k}_{2}$ электрон, отдавая свою энергию соседнему электрону, рекомбинируется $\left(k_{2}^{\prime}\right)$ с дыркой валентной зоны.

До процесса рекомбинации энергия электронов имеет следующий вид:

$$
E_{c}\left(k_{1}\right)=E_{g}+\frac{\hbar^{2} k_{1}^{2}}{2 m_{n}}, E_{c}\left(k_{2}\right)=E_{g}+\frac{\hbar^{2} k_{2}^{2}}{2 m_{n}}
$$

После процесса рекомбинации энергия электронов выглядит так:

$$
E_{c}\left(k_{1}^{\prime}\right)=E_{g}+\frac{\hbar^{2} k_{1}^{\prime 2}}{2 m_{n}}, \quad E_{V}\left(k_{2}^{\prime}\right)=-\frac{\hbar^{2} k_{2}^{\prime 2}}{2 m_{p}}
$$




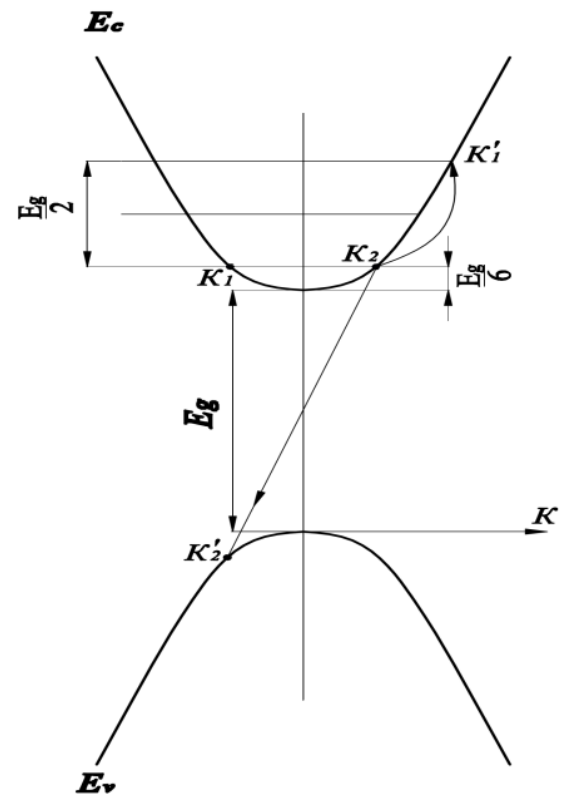

$a$

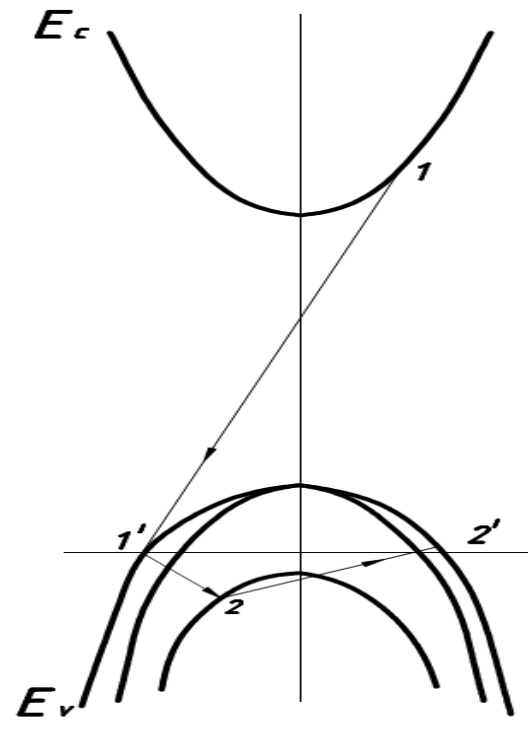

6

Рис.1. Прочессы Оже-рекомбинации в прямозонных полупроводниках

В данном случае закон сохранения энергии записывается следующим образом:

$$
2 E_{g}+\frac{\hbar^{2} k_{1}^{2}}{2 m_{n}}+\frac{\hbar^{2} k_{2}^{2}}{2 m_{n}}=E_{g}+\frac{\hbar^{2} k_{1}^{2}}{2 m_{n}}-\frac{\hbar^{2} k_{2}^{2}}{2 m_{p}}
$$

А закон сохранения импульса

$$
k_{1}+k_{2}=\grave{k_{1}}+\grave{k_{2}}
$$

Вероятность пребывания электронов в зоне проводимости в $k_{1}$

$$
\begin{aligned}
f\left(E_{c}\left(k_{1}\right)\right) & =\frac{n}{N_{c}} e^{-\frac{\hbar^{2} k_{1}^{2}}{2 m_{n} k_{0} T}} \\
f\left(E_{V}\left(k_{2}\right)\right) & =\frac{p}{N_{V}} e^{-\frac{\hbar^{2} k_{2}^{2}}{2 m_{p} k_{0} T}}
\end{aligned}
$$

Использовав данные выражения, получаем следующее

$$
f\left(E _ { c } ( k _ { 1 } ) f ( E _ { c } ( k _ { 2 } ) ) \cdot \left(1-f\left(E_{V}\left(k_{2}^{\prime}\right)\right)=\frac{n^{2} p}{N_{c}^{2} N_{V}} \exp \left\{-\left[\frac{\hbar^{2}\left(k_{1}^{2}+k_{2}^{2}\right)}{2 m_{n}}+\frac{\hbar^{2} k_{2}^{2}}{2 m_{p}}\right] / k_{0} T\right\}\right.\right.
$$

При условии $m_{n}=m_{p}$ и $\grave{k_{2}} \|\left(k_{1}+k_{2}\right), \grave{k_{2}}=\frac{k_{1} k_{2}-\frac{E g m_{n}}{\hbar^{2}}}{\left|k_{1}+k_{2}\right|^{2}}\left(k_{1}^{2}-k_{2}^{2}\right)$ согласно отношению (6) скорость Оже-рекомбинации происходит с наиболее высокой вероятностью.

$$
k_{1}=k_{2}=k_{0}, \quad\left|k_{0}\right|=\frac{1}{3}\left(\frac{E_{g} m_{n}}{\hbar^{2}}\right)^{1 / 2} \text { является условием для максимального достижения }
$$
выражения (6). Применяя данные отношения выражение (6) можно записать следующим образом

$$
f\left(E _ { c } ( k _ { 1 } ) f ( E _ { c } ( k _ { 2 } ) ) \cdot \left(1-f\left(E_{V}\left(k_{2}^{\prime}\right)\right)=\frac{n^{2} p}{N_{c} N_{V}} \exp \left(-\frac{E_{g}}{2 k_{0} T}\right)\right.\right.
$$

При этом в рекомбинации принимают участие электроны, расположенные выше $E_{g} / 2$.

Если $m_{n} \neq m_{p}, E\left(k_{1}\right)=E\left(k_{2}\right)>E_{c o}$, то $\mathrm{E}_{1}=\mathrm{E}_{2}=\mathrm{E}_{\mathrm{co}}+\frac{\mathrm{E}_{\mathrm{g}} \theta^{2}}{\left(1+3 \theta+2 \theta^{2}\right)}$, при этом $\theta=\frac{m_{n}}{m_{p}}$, в данном состоянии $E_{V O}-E_{2}=\frac{E_{g} \theta}{1+3 \theta+2 \theta^{2}}$ рекомбинации принимают участие электроны, расположенные выше $E_{g} / 6$.

Если $m_{n}=m_{p}$; то $E_{1}=E_{2}=E_{C}+\frac{E_{g}}{6}$, при $m_{n} \ll m_{p}$ становится $E_{1}=E_{2}=E_{C O}$. При этом состоянии в Оже-рекомбинации участвуют электроны верхней валентной зоны и нижней зоны проводимости. В положении термодинамического равновесияв Оже-рекомбинации принимают участие 2 электрона и 1 дырка. Скорость рекомбинации при этом выражается 
Здесь $I_{1}=\int u_{C}^{*}\left(k_{1} r\right) u_{V}\left(\grave{k_{1}} r\right) d^{3} r$

$$
r=\frac{8 \pi^{2} \beta e^{4} \hbar^{2}\left|I_{1} I_{2}\right|^{2} \theta^{3 / 2} n^{2} p \exp \left(-E_{g} \theta /(1+\theta) k_{0} T\right.}{(4 \pi \varepsilon)^{2} m_{n}^{2}\left(k_{0} T\right)^{2} E_{g}(1+\theta) \sqrt{2(1+2 \theta)}}
$$

$I_{2}=\int u_{V}^{*}\left(k_{2} r\right) u_{C}\left(k_{2} r\right) d^{3} r$ интгерал функции Блоха.

Оценив выражение (8), если $\beta=3, \theta=1,\left|I_{1} \cdot I_{2}\right| \approx 10^{-2}$, коэффициент рекомбинации равен $\mathrm{c}_{n}=10^{-28}\left(\frac{10}{\varepsilon}\right)\left(\frac{m_{o}}{m_{n}}\right)^{2}\left(\frac{1 e V}{E_{g}}\right)\left(\frac{300}{T}\right) e^{-\frac{E g}{2 k_{0} T}}$.

Для $\mathrm{InSb}$ при $E_{g}=0,3 \mathrm{eV}, m_{n}=m_{p}, \quad$ и $n=10^{15} \mathrm{~cm}^{-3}, \mathrm{c}_{n}=10^{-30} \mathrm{~cm}^{6} /$ сек. Время жизни носителей заряда равно

$$
\tau=\left(\frac{E_{g}}{k_{0} T}\right) \exp \left(\frac{(1+2 \theta) E_{g}}{(1+\theta) k_{0} T}\right) .
$$

Теперь рассмотрим процесс Оже-рекомбинации в сложнозонных полупроводниках. Известно, что полупроводники $\mathrm{Si}, \mathrm{Ge}, \mathrm{AsGa}$ относятся к сложнозонным полупроводникам, так как их валентнные зоны состоят из трёх сетей. ( Рис.2).

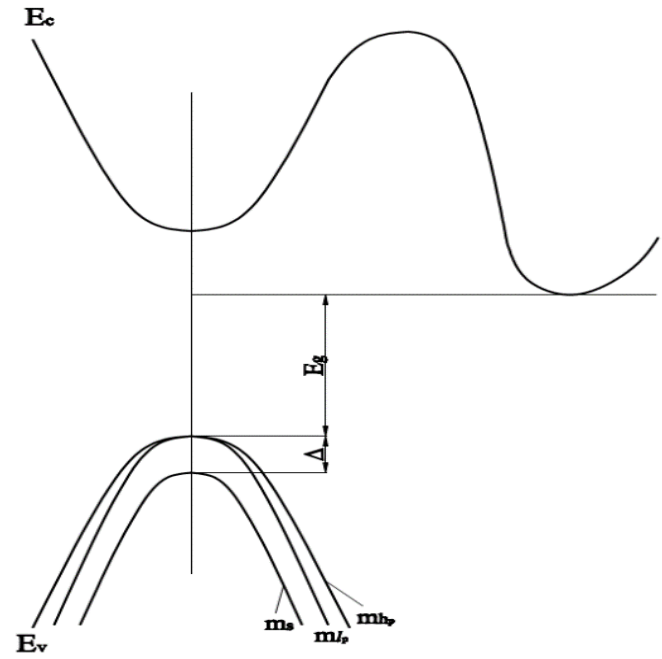

Рис.2. Оже-рекомбинация в сложнозонных подупроводниках

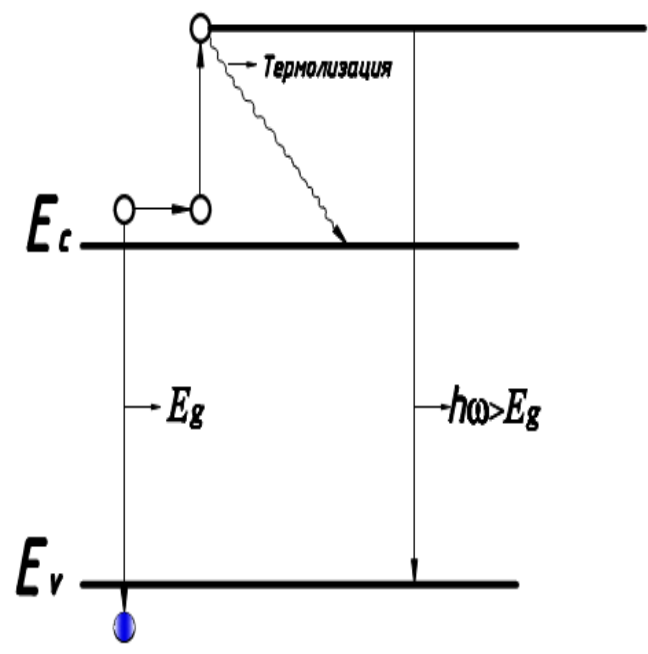

Рис.3. Один вид Оже-рекомбинащии

В данном случае процессы рекомбинации зависят от отношения дистанции $\Delta$ между шириной запрещённой зоны полупроводника $E_{g}$ и сетей валентной зоны.

Если $\Delta>E_{g}$, взаимная оптическая проводимость между сетями электронов почти не вносит вклад в Оже-рекомбинацию. При $\Delta=E_{g}$, проводимость электронов между сетями вносит большой вклад в Оже-рекомбинацию.

В прямозонных полупроводниках Оже-рекомбинация происходит следующим образом (Рис.1б). Первый электрон рекомбинирует с 1-й дыркой, 1 -я дырка передаёт свою лишнюю энергию 2-й дырке и она может перейти в состояние 2'. При этом требуемая минимальная энергия равна

$$
E_{\min }=\frac{2 m_{n p}+m_{n}^{*}}{2 m_{h p}+m_{n}^{*}-m_{s}}\left(E_{g}-\Delta\right)
$$

В связи со значением переменной $E_{g}-\Delta$ энергия активизации Оже рекомбинации будет в промежутке $0<E \leq \frac{E_{g}}{2}$.

Таким образом, в Оже-рекомбинации наблюдались следующие физические явления.

1. В процессе Оже-рекомбинации с повышением концентрации основных носителей заряда уменьшается время жизни неосновных. В выражении $\tau_{p}=\frac{1}{n}, \tau_{n}=\frac{1}{p}$ для Ge типа $n$ при $n=10^{17} \mathrm{~cm}^{-3}$, равно $\tau_{n}=50-60$ мкс. , $\tau_{p}=1$ мкс.. 
2. $\tau \sim \frac{1}{\mathrm{~T}},-$ то есть с повышением температуры время жизни носителей заряда уменьшается.

3. С внесением в полупроводниковые материалы примесей скорость рекомбинации резко возрастает, то есть $\mathrm{r}=\frac{\Delta n}{\tau_{n}}$,

4. При уменьшении концентрации носителей заряда каким-либо способом, снижается скорость излучательной рекомбинации, что показывает умеьшение интенсивности излучения $r_{\text {излуч }}=\gamma_{n} n p$. При уменьшении концентрации снижение интенсивности излучения называется люминесцентно-концентрационным снижением. С повышением температуры повышается кинетическая энергия электронов, при этом наблюдается повышение вероятности Ожерекомбинации.

5. В полупроводниках с непрямыми зонами скорость рекомбинации бывает относительно ниже прямозонных полупроводников.

6. Электрон, вступивший в рекомбинацию с дыркой, передаёт свою лишнюю энергию соседнему электрону. Электроны, получившие лишнюю энергию, называются горячими электронами и они в течение короткого времени передают свою энергию кристаллической решётке, это явление называется термолизацией (Рис. 3) для

$$
2 E_{g}>h \omega_{\text {нур }} \geq E_{g}, \quad h \omega_{\text {нур }} \approx 2 E_{g}(\mathrm{Ge})
$$

\section{ЛИТЕРАТУРА}

1. Бочкараева Н.И., Ребане Ю.Т., Шритер Ю.Г. Рост скорости рекомбинации Шокли-Рида-Холла в квантовых ямах InGaN/GaN как основной механизм падения эффективности светодиодов при высоких уровнях инжекции. //Физика и техника полупроводников, 2015, том 49, вып. 12, с.1714-1719.

2. Dyakonov M.I., Kachorovskii V.Y. Nonthreshold Auger recombination in quantum wells //Phys.Rev. B.American Physical Society, 1994. Vol. 49. №24. P.17130-17138.

3. Krishnamurthy S., Berding M.A. Yu.Z.G. Minority carrier lifetimes in HgCdTe alloys// J/Electron. Mater.Springer-Verlag, 2006. Vol.35, №6, P.1369-1378.

4. Casselman T.N., Petersen P.E. A comparison of the dominant Auger transitions in p-type $(\mathrm{Hg}, \mathrm{Cd}) \mathrm{Te} / /$ Solid State Commun. 1980. Vol.33, №6, P.615-619.

5. Iveland J., Piccardo M., Martinelly L., Peretti J., ChoiJ.W., Young N., Nakamura S., Speck J.S., Weisbuch C. //Appl.Phus.Lett.,105,052103(2014).

6. Яковлева Н.И. Механизмы Оже-рекомбинации в узкозонных полупроводниковых структурах HgCdTe. //Успехи прикладной физики, 2018, том 6, №2, с.130-139.

7. Винниченко М.Я., Махов И.С., Селиванов А.В., Сорокина А.М., Воробьев Л.Е., Фирсов Д.А., Штеренгас Л.М., Беленький Г.Л. Влияние Оже-рекомбинации на концентрацию неравновесных носителей заряда в квантовых ямах InGaAsSb/AlGaAsSb.

8. Аликулов М.Н. Зависимость структуры зон полупроводников от скорости рекомбинации между зонами. World science № 5(57) Vol. 1, Мау 2020, стр. 31-34. 